Bull. Austral. Math. Soc.

VOL. 47 (1993) [395-406]

\title{
LOCALLY COMPACTLY LIPSCHITZIAN MAPPINGS IN INFINITE DIMENSIONAL PROGRAMMING
}

\author{
B.M. GLover
}

In this note we show that a subgradient multifunction of a locally compactly Lipschitzian mapping satisfies a closure condition used extensively in optimisation theory. In addition we derive a chain rule applicable in either separable or reflexive Banach spaces for the class of locally compactly Lipschitzian mappings using a recently derived generalised Jacobian. We apply these results to the derivation of Karush-Kuhn-Tucker and Fritz John optimality conditions for general abstract cone-constrained programming problems. A discussion of constraint qualifications is undertaken in this setting.

\section{INTRODUCTION}

Recently several authors have considered the problem of deriving optimality conditions for abstract programming problems involving locally Lipschitz functions (see [2, $6,10,15])$. In the main, the tool used in these proofs has been Ekeland's Theorem [9] and, without exception, some closedness condition is required on a subgradient multifunction. In this paper we establish that a large class of locally Lipschitz mappings satisfy the required type of closedness. These mappings are called locally compactly Lipschitzian, and were first developed, as an attempt to extend the nonsmooth analysis of Clarke [3] to the vector-valued setting, in the pioneering work of Thibault (see [19, $20,21])$. As an important consequence of this result we are able to derive an infinite dimensional chain rule for these functions valid in either separable or reflexive Banach spaces, thus extending the partially finite dimensional result due to Clarke [3]. This result will be particularly useful as a tool in the derivation of optimality conditions, via exact penalty techniques, for programming problems in infinite dimensional spaces. As another immediate consequence of this we consider the problem of deriving KarushKuhn-Tucker (KKT) type optimality conditions involving explicit Lagrange multipliers for locally compactly Lipschitzian programming problems in separable spaces. This extends the recent work of Flam [10]. In addition we develop a new Robinson-type regularity condition for nonsmooth mappings using a generalised Jacobian valid in reflexive Banach spaces; this condition is compared with existing regularity conditions.

Received 30th June, 1992

The author would like to thank Dr. Danny Ralph for suggesting the use of locally compactly Lipschitzian mappings in the contert of Theorem 1 and Dr. Ewan Barker for a lively discussion of several topological questions related to this paper.

Copyright Clearance Centre, Inc. Serial-fee code: 0004-9729/93 SA2.00+0.00. 


\section{Main Results}

Throughout we shall assume that $X$ and $Y$ are Banach spaces. Reflexivity or separability of $Y$ will only be required where it is explicitly stated. The remaining terminology to be used is standard in the field of nonsmooth analysis (see Clarke [3]). We begin with some preliminary definitions.

Definition: [21] A mapping $f: X \rightarrow Y$ is said to be (strongly) compactly Lipschitzian at a point $\bar{x} \in X$ if there is a mapping $K$ from $X$ into the $\operatorname{set} \operatorname{Comp}(Y)$ of nonempty (strongly) compact subsets of $Y$, a mapping $r$ of $(0,1] \times X \times X$ into $Y$ and neighbourhoods $U$ of $\bar{x}$ and $V$ of zero in $X$ satisfying:

(a) $\lim _{t \downarrow 0, x \rightarrow \bar{x}} r(t, x ; v)=0$ for each $v \in X$ and $\lim _{t \downarrow 0, x \rightarrow \bar{x}, v \rightarrow 0} r(t, x ; v)=0$;

(b) for all $x \in U, v \in V$ and $t \in(0,1]$

$$
t^{-1}[f(x+t v)-f(x)] \in K(v)+r(t, x ; v) ;
$$

(c) $K(0)=\{0\}$ and the set set-valued mapping $K$ is upper semi-continuous at the origin (that is, for every neighbourhood $W$ of $K(0)$ in $Y$ there is a neighbourhood $L$ of zero in $X$ satisfying $K(v) \subseteq W$ for every $v \in L)$.

If $f$ is compactly Lipschitzian at every $x \in X$ then $f$ will be said to be locally compactly Lipschitzian.

It can be shown ([19]) that every locally compactly Lipschitzian mapping is locally Lipschitz in the usual sense. In finite dimensions the concepts coincide. For a discussion of the properties of these mappings and examples in the infinite dimensional setting the reader is referred to [19] and [20].

Recently Ralph [16] has developed a concept of generalised Jacobian applicable to locally Lipschitz mappings $f: X \rightarrow Y$ where $X$ is a Banach space and $Y$ is a reflexive Banach space. Namely, the generalised Jacobian of $f$ at $a$ is given by:

$$
\partial f(a)=\left\{A \in L(X, Y):\left(\forall(\lambda, u) \in Y^{\prime} \times X\right) \lambda A(u) \leqslant(\lambda f)^{\circ}(a, u)\right\} .
$$

We shall use $\partial f(a)$ to denote both the Clarke subgradient when $f$ is real-valued and the generalised Jacobian when $f$ is vector-valued as no confusion should arise. Here $(\lambda f)^{\circ}(a, u)$ denotes the Clarke generalised directional derivative of the real-valued function $\lambda f$ at $a$ in the direction $u$. In [16] it is shown that $\partial f(a)$ is nonempty, convex and weak operator compact and, in particular, $\partial(\lambda f)(a)=\lambda \partial f(a)$ for every $\lambda \in Y^{\prime}$. For other properties the reader is referred to [16]. The idea of a subgradient for a locally Lipschitz mapping leads immediately to concepts of closure for the multifunction $(\lambda, x) \rightarrow \partial(\lambda f)(x)$. The closure of this subgradient multifunction is of course related to the upper semi-continuity of the function $(\lambda, x, u) \rightarrow(\lambda f)^{\circ}(x, u)$. In this 
paper we shall concentrate on closure for convenience. In particular we shall say this subgradient multifunction is weak ${ }^{*}$ closed if for sequences $\lambda_{i} \rightarrow \lambda, w_{i} \rightarrow w, x_{i} \rightarrow x$ with $w_{i} \in \partial\left(\lambda_{i} f\right)\left(x_{i}\right)$ it follows that $w \in \partial(\lambda f)(x)$. Of course the convergence here is very important. We shall assume that sequences in the dual spaces $X^{\prime}$ and $Y^{\prime}$ are converging in the weak* topology and other sequences converging strongly (in the norm topology). Note in [16] it is shown that if the convergence of $\left(\lambda_{i}\right)$ is taken in the strong topology of $Y^{\prime}$ then every locally Lipschitz mapping will possess a subgradient multifunction with this type of closure. The following theorem shows the connection with locally compactly Lipschitzian mappings.

THEOREM 1. Let $f: X \rightarrow Y$ be locally compactly Lipschitzian. Then the mapping $(\lambda, x) \rightarrow \partial(\lambda f)(x)$ is weak* closed.

PROOF: Let $\lambda_{i} \stackrel{w^{*}}{\rightarrow} \bar{\lambda}, x_{i} \rightarrow \bar{x}, w_{i} \stackrel{w^{*}}{\rightarrow} \bar{w}$ with $w_{i} \in \partial\left(\lambda_{i} f\right)\left(x_{i}\right)$ for each $i$. Now fix $\varepsilon>0, v \in V$, and $i$, then

$$
\begin{aligned}
w_{i}(v) & \leqslant\left(\lambda_{i} f\right)^{\circ}\left(x_{i}, v\right) \\
& =\lim _{j}\left(\frac{\lambda_{i} f\left(x_{i}^{j}+t_{i}^{j} v\right)-\lambda_{i} f\left(x_{i}^{j}\right)}{t_{i}^{j}}\right)
\end{aligned}
$$

for some sequences $t_{i}^{j} \downarrow 0$ and $x_{i}^{j} \rightarrow x_{i}$.

$$
\leqslant \frac{\lambda_{i} f\left(x_{i}^{j}+t_{i}^{j} v\right)-\lambda_{i} f\left(x_{i}^{j}\right)}{t_{i}^{j}}+\varepsilon
$$

for $j$ sufficiently large, say $j>N_{i}(\varepsilon)$.

We now select, for each $i, j=j(i, \varepsilon)$ such that $j>N_{i}(\varepsilon),\left\|x_{i}^{j}-x_{i}\right\|<1 / i, 0<$ $t_{i}^{j}<1 / i$. This is possible since $x_{i}^{j} \rightarrow x_{i}$ and $t_{i}^{j} \downarrow 0$ as $j \rightarrow \infty$. Let $y_{i}=x_{i}^{j(i, e)}$ and $\alpha_{i}=t_{i}^{j(i, \varepsilon)}$ for each $i$. Clearly, as $x_{i} \rightarrow \bar{x}$, we have $y_{i} \rightarrow \bar{x}$ and $\alpha_{i} \downarrow 0$ with, for each $i$,

$$
w_{i}(v) \leqslant \frac{\lambda_{i} f\left(y_{i}+\alpha_{i} v\right)-\lambda_{i} f\left(y_{i}\right)}{\alpha_{i}}+\varepsilon .
$$

Now since $f$ is locally compactly Lipschitzian it follows, by taking a subsequence if necessary, that

$$
\frac{f\left(y_{i}+\alpha_{i} v\right)-f\left(y_{i}\right)}{\alpha_{i}} \rightarrow q
$$

for some $q \in K(v)$. For convenience let

$$
q(x, t ; v)=\frac{f(x+t v)-f(x)}{t} .
$$


Then,

$$
\begin{aligned}
\bar{w}(v)= & \lim _{i} w_{i}(v) \\
\leqslant & \lim _{i} \lambda_{i}\left(q\left(y_{i}, \alpha_{i} ; v\right)\right)+\varepsilon \\
= & \lim _{i}\left[\lambda_{i}\left(q\left(y_{i}, \alpha_{i} ; v\right)-q\right)+\lambda_{i}(q)\right]+\varepsilon \\
\leqslant & \lim _{i} k\left\|q\left(y_{i}, \alpha_{i} ; v\right)-q\right\|+\bar{\lambda}(q)+\varepsilon \\
& \text { where } \left.\left(\lambda_{i}\right) \text { is norm-bounded (by } k\right) \text { using the uniform } \\
& \text { boundedness theorem as } Y \text { is a Banach space and the } \\
& \text { sequence is weak* convergent (see [23, p.69]) } \\
= & \bar{\lambda}(q)+\varepsilon \\
= & \bar{\lambda}\left(\lim _{i} q\left(y_{i}, \alpha_{i} ; v\right)\right)+\varepsilon \\
= & \lim _{i} \bar{\lambda}\left(q\left(y_{i}, \alpha_{i} ; v\right)\right)+\varepsilon \\
\leqslant & \limsup _{x \rightarrow \bar{x}, t \downarrow 0} \frac{\bar{\lambda} f(x+t v)-\bar{\lambda} f(x)}{t}+\varepsilon \\
= & (\bar{\lambda} f)^{\circ}(\bar{x}, v)+\varepsilon .
\end{aligned}
$$

Thus, since $\varepsilon$ was arbitrary, we have $\bar{w}(v) \leqslant(\bar{\lambda} f)^{\circ}(\bar{x}, v)$ for all $v \in V$. Thus $\bar{w} \in$ $\partial(\bar{\lambda} f)(\bar{x})$ (since $V$ is a neighbourhood of zero and using the positive homogeneity of $\bar{w}(\cdot)$ and $\left.(\bar{\lambda} f)^{\circ}(\bar{x}, \cdot)\right)$. Thus the required mapping is weak* closed.

REMARK. We can now derive an infinite dimensional chain rule which is directly applicable to locally compactly Lipschitzian functions. The approach we shall use will require, critically, that a norm-bounded sequence in the dual space $Y^{\prime}$ has a convergent subsequence. It is an immediate corollary of the Banach-Alaoglu Theorem that a norm-bounded sequence will possess a convergent subnet in $Y^{\prime}$. However to ensure this subnet is actually a subsequence we require either separability or reflexivity of $Y$.

Theorem 2. Let $X$ and $Y$ be Banach spaces with $Y$ either reflexive or separable. Let $f: X \rightarrow Y$ be locally Lipschitz with the mapping $(\lambda, x) \rightarrow \partial(\lambda f)(x)$ weak $^{*}$ closed. Let $a \in X$ and let $g: Y \rightarrow \mathbb{R}$ be locally Lipschitz at $f(a)$. Then $g \circ f$ is locally Lipschitz at $a$ and

$$
\partial(g \circ f)(a) \subseteq \text { weak }^{*} \text {-clco } \bigcup_{\lambda \in \partial g(f(a))} \partial(\lambda f)(a) .
$$

Furthermore if $Y$ is reflexive then this condition becomes

$$
\partial(g \circ f)(a) \subseteq \text { weak }^{*}-c l c o[\partial g(f(a)) \circ \partial f(a)]
$$


Proof: Clearly $g \circ f$ is locally Lipschitz. Let $v \in X$ and consider the following:

$$
\alpha_{v}=\max \{w(v): w \in \partial(\lambda f)(a), \lambda \in \partial g(f(a))\} \text {. }
$$

The result will follow if we can show that $(g \circ f)^{\circ}(a, v) \leqslant \alpha_{v}$. Now, there are sequences $\left(x_{i}\right) \subset X, x_{i} \rightarrow a$ and $\left(t_{i}\right) \subset \mathbb{R}_{+}, t_{i} \downarrow 0$ such that

$$
(g \circ f)^{\circ}(a, v)=\lim _{i} \frac{g \circ f\left(x_{i}+t_{i} v\right)-g \circ f\left(x_{i}\right)}{t_{i}} .
$$

Consider the quotient

$$
\frac{g \circ f\left(x_{i}+t_{i} v\right)-g \circ f\left(x_{i}\right)}{t_{i}}=\frac{\lambda_{i} f\left(x_{i}+t_{i} v\right)-\lambda_{i} f\left(x_{i}\right)}{t_{i}}
$$

for some $\lambda_{i} \in \partial g\left(z_{i}\right)$ where $z_{i}$ lies on the line segment joining $f\left(x_{i}+t_{i} v\right)$ and $f\left(x_{i}\right)$ (this follows by a direct application of the Lebourg mean-value theorem (see [3])). Note that $z_{i} \rightarrow f(a)$ by continuity of $f$. Also the sequence $\left(\lambda_{i}\right)$ is norm-bounded (by the Lipschitz constant of $g$ locally about $f(a)$ ). Hence, by using a subsequence (since $Y$ is either separable or reflexive) if necessary, it follows that $\left(\lambda_{i}\right)$ converges (weak*) to some $\bar{\lambda} \in Y^{\prime}$. In addition, using the strong closure of the subgradient multifunction of $g$, it follows that $\bar{\lambda} \in \partial g(f(a))$. Now, we again apply the mean-value theorem to obtain

$$
\frac{\lambda_{i} f\left(x_{i}+t_{i} v\right)-\lambda_{i} f\left(x_{i}\right)}{t_{i}}=w_{i}(v)
$$

for some $w_{i} \in \partial\left(\lambda_{i} f\right)\left(y_{i}\right)$ where $y_{i}$ lies on the line segment connecting $x_{i}+t_{i} v$ and $x_{i}$. Hence $y_{i}$ converges to $a$. Also since $\left(\lambda_{i}\right)$ is norm-bounded and $f$ is locally Lipschitz it follows that the sequence $\left(w_{i}\right)$ is also norm-bounded. We can therefore, by using a subsequence if necessary, assume that $\left(w_{i}\right)$ converges to $\bar{w}$. Now applying the weak* closure of the subgradient multifunction it follows that $\bar{w} \in \partial(\bar{\lambda} f)(a)$ with $\bar{\lambda} \in \partial g(f(a))$. Hence

$$
\begin{aligned}
(g \circ f)^{\circ}(a, v) & =\lim _{i} \frac{g \circ f\left(x_{i}+t_{i} v\right)-g \circ f\left(x_{i}\right)}{t_{i}} \\
& =\lim _{i} \frac{\lambda_{i} f\left(x_{i}+t_{i} v\right)-\lambda_{i} f\left(x_{i}\right)}{t_{i}} \\
& =\lim _{i} w_{i}(v) \\
& =\bar{w}(v) \\
& \leqslant(\lambda f)^{\circ}(a, v) \\
& \leqslant \alpha_{v} .
\end{aligned}
$$

Thus the result follows since $v$ was chosen arbitrarily.

The final statement follows easily since $\partial(\lambda f)(a)=\lambda \partial f(a)$ using the generalised Jacobian. 
REMARK. This result can be extended to the case in which $g: Y \rightarrow W$ with $W$ a reflexive Banach space along the lines of a similar extension in [16]. Using Theorem 1 and 2 above we can derive the following corollary.

COROLLARY 1. The result of Theorem 2 remains valid if $f$ is assumed locally compactly Lipschitzian and $g$ is locally Lipschitz.

Proof: Immediate by Theorem 1 above.

EXAMPLE. Consider the special case of Theorems 1 and 2 in which $f$ is locally compactly Lipschitzian, and $g$ is defined as follows:

$$
g(x)=d(x,-S)=\inf \{\|x+s\|: s \in S\}
$$

where $S \subseteq Y$ is a closed convex cone. Assume, additionally, that $f(a) \in-S$. Then it follows that

$$
\partial(g \circ f)(a) \subseteq c l c o\left\{v: v \in \partial(\lambda f)(a), \lambda \in S^{*},\|\lambda\| \leqslant 1, \lambda f(a)=0\right\} .
$$

This is clear since $g$ is a continuous convex function, hence it is locally Lipschitz. It is well known that the convex subdifferential of $g$ at a point $y \in-S$ is given by:

$$
\partial g(y)=\left\{v \in S^{*}:\|v\| \leqslant 1, v(y)=0\right\} .
$$

Thus the result follows by a direct application of Corollary 1 .

REMARK. Note that the above result provides a chain rule which would be immediately applicable to abstract optimisation problems involving cone constraints (as discussed in the next section) where optimality conditions are sought via exact penalty techniques; see Burke [2] for an excellent survey of this approach. Note that, assuming reflexivity of $Y$, we could extend this example using the generalised Jacobian. It should be noted that Dien [5] has derived a version of this example in the more general setting of Lipschitzian set-valued mappings in Hilbert space (see also Dien and Sach [8] where the result is used in establishing metric regularity results in the multifunction setting).

\section{Application to Optimisation}

In this section we shall consider the derivation of optimality conditions for the following abstract cone-constrained programming problem:

$$
\begin{aligned}
& \text { (P) Minimise } h(x) \\
& \text { subject to }-f(x) \in S, x \in C
\end{aligned}
$$


where $h: X \rightarrow \mathbb{R}, f: X \rightarrow Y, X$ and $Y$ are Banach spaces with $Y$ separable; $S \subseteq Y$ is a closed convex cone with nonempty interior and $C$ is a closed set. Note that the dual cone to $S$ will be denoted by $S^{*}=\left\{\mu \in Y^{\prime}:(\forall s \in S) \mu(s) \geqslant 0\right\}$, the Clarke tangent cone to $C$ at $a$ is $T_{C}(a)$ (see [3]) and the normal cone to $C$ at $a$ is $N_{C}(a)=-\left(T_{C}(a)\right)^{*}$ in our terminology (see [3]).

We begin by noting that Flàm [10] has recently obtained a Fritz John type optimality condition for (P) in the separable Banach space setting (see Theorem 3 below). The proof relied critically on the Clarkson-Riefel renorming lemma (see [12]), and so is restricted to the separable setting, as well as the weak* closure of the subgradient multifunction. The method of proof was similar to the finite dimensional approach, employing Ekeland's Theorem [9], used by Clarke [3]. This result corrects the main theorem in Lai [15] where the strengthened closure of the subgradient multifunction was overlooked. A similar result, in the Hilbert space setting, has been derived by Dien [5] and [6] in the context of set-valued constraints under the assumption of the $\mathrm{Cl}$-property which reduces to weak* closure of the subgradient multifunction for singlevalued mappings.

THEOREM 3. For problem $(P)$ assume $h$ and $f$ are locally Lipschitz with the mapping $(\lambda, x) \rightarrow \partial(\lambda f)(x)$ weak $^{*}$ closed. Then if $a \in X$ is a local solution to $(P)$ there exists $(0,0) \neq(\tau, \lambda) \in \mathbb{R}_{+} \times S^{*}$ such that

$$
0 \in \tau \partial h(a)+\partial(\lambda f)(a)+N_{C}(a), \lambda f(a)=0 .
$$

If, in addition, we assume that $Y$ is reflexive then (1) above becomes (using the generalised Jacobian):

$$
0 \in \tau \partial h(a)+\lambda \partial f(a)+N_{C}(a), \lambda f(a)=0 .
$$

Proof: Follows directly by [10, Theorem 3.2]. The final comment follows since, for any $\lambda \in Y^{\prime}, \partial(\lambda f)(a)=\lambda \partial f(a)$ (see [16] for details).

REMARK. It should be noted that, by Theorem 1, the result of Theorem 3 remains valid when $f$ is locally compactly Lipschitzian. It remains to consider now regularity conditions which will ensure that in the Fritz John Theorem above the multiplier $\tau$ is positive. Various conditions have been used to ensure that Karush-Kuhn-Tucker conditions (KKT) are established. This is of particular importance in the stability analysis of the program. Below we shall present an extension of the Robinson regularity condition [17].

Definition: Let $f: X \rightarrow Y$ be locally Lipschitz with $Y$ reflexive. We shall say that the constraint system $-f(x) \in S, x \in C$ satisfies the generalised Robinson regularity condition (RRC) at $a \in X$ if

$$
(\forall A \in \partial f(a)) 0 \in \operatorname{int}\left(f(a)+A\left(T_{C}(a)\right)+S\right) .
$$


Using the generalised open mapping theorem (see [24]) it can be shown, under the additional assumptions that $C$ is a closed convex set with $a \in C$ and $f(a) \in-S$, that (2) implies the following

$$
(\forall A \in \partial f(a))(\exists \rho>0) Y_{\rho} \subseteq\left(A\left((C-a) \cap X_{1}\right)+(S+f(a)) \cap Y_{1}\right)
$$

where $Y_{\rho}$ denotes the closed ball in $Y$ centred at the origin of radius $\rho$, similarly $X_{1}, Y_{1}$ denote the closed unit balls in $X$ and $Y$ respectively. It should be noted that if $f$ is continuously Fréchet differentiable then (2) reduces to the well known Robinson regularity condition (see [17]).

For the case in which $C$ is a closed convex set we shall say the constraint system satisfies the uniform generalised Robinson regularity condition (URRC) at $a$ if (3) holds uniformly for each $A \in \partial f(a)$ (in the sense that $\rho$ is independent of $A$ ). Note that Yen [22] has recently shown, in the finite dimensional setting using the Clarke generalised Jacobian, that (2) is sufficient for metric regularity (see $[1,13,14]$ ) of the constraint system. Namely, the constraint system satisfies the metric regularity condition at $a \in X$ if

$$
(\exists \gamma>0)(\exists U \subseteq X, 0 \in \text { int } U)(\forall x \in U \cap C) d(x, F) \leqslant \gamma d(f(x),-S)
$$

where $F=\{x \in C: f(x) \in-S\}$. It is well known that metric regularity implies calmness of $(\mathrm{P})$. This regularity condition and its connection with exact penalty techniques has been detailed in Burke [2] (see also Sach [18]).

Another sufficient condition for metric regularity, applicable to nonsmooth mappings in infinite dimensional spaces, which has been used by several authors (see [6, 7]), is the following. The point $a \in X$ will be said to be a regular point of the constraint system $f(x) \in-S, x \in C$ if there exist positive constants $\gamma, \beta$ and a neighbourhood $U$ of $a$ such that for each $x \in U \cap C, \lambda \in S^{*}$ (with $\|\lambda\|=1$ ) and $w \in \partial(\lambda f)(x)$ there is $u \in T_{C}(x) \cap X_{1}$ satisfying

$$
-\lambda f(x)-\beta w(u) \geqslant \gamma
$$

This condition was stated more generally for multifunctions in [8]; we have restricted it to the single-valued case for our purposes. Note that under this regularity condition and weak* closure of the subgradient multifunction Dien and Yen [7] have derived an implicit function theorem which, essentially, establishes metric regularity of the constraint system in (P). Consequently using Theorem 1 of this paper the results in [7], particularly Corollary 2.2, would be applicable under the regularity condition (4) above. We shall now show, under suitable upper semi-continuity assumptions, that (URRC) is sufficient for $a$ to be regular point of the constraint system in the infinite dimensional setting. Consequently, by [7, Corollary 2.2], (URRC) is sufficient for metric regularity. 
Definition: A multifunction $\Gamma: X \rightarrow 2^{X}$ is said to be strongly upper semicontinuous at $a \in X$ if, for each $\varepsilon>0$ there is a $\delta>0$ such that whenever $\|x-a\|<\delta$ we have $\Gamma(x) \subseteq \Gamma(a)+\varepsilon X_{1}$.

THEOREM 4. For the constraint system of $(P)$ assume $f: X \rightarrow Y$ is locally Lipschitz with $Y$ reflexive and $C$ a closed convex set. Furthermore assume the generalised Jacobian multifunction $x \rightarrow \partial f(x)$ is strongly upper semi-continuous at the feasible point $a \in X$, and that the system satisfies (URRC) there. Then $a$ is a regular point for $(P)$.

Proof: Assume that (URRC) is satisfied at a. Hence there exists $\rho>0$ such that for each $A \in \partial f(a)$,

$$
Y_{\rho} \subseteq\left(A\left((C-a) \cap X_{1}\right)\right)+(f(a)+S) \cap Y_{1} .
$$

Now let $D(A)=A\left((C-a) \cap X_{1}\right)+(f(a)+S) \cap Y_{1}$, then by $[24$, Lemma 5.1] it follows that

$$
\sigma(\mu, D(A)) \geqslant \rho
$$

for each $\mu \in Y^{\prime}$ with $\|\mu\|=1$. Here, for a set $E \subseteq Y$, we denote the support functional of $E$ by $\sigma(\mu, E)=\sup \{\mu(e): e \in E\}$, for each $\mu \in Y^{\prime}$. It immediately follows, from (5), that for each $\lambda \in S^{*}$ with $\|\lambda\|=1$ there are $u \in(C-a) \cap X_{1}$ and $s \in S$ such that

$$
\begin{gathered}
-\lambda A(u)-\lambda f(a)-\lambda(s) \geqslant \frac{\rho}{2} \\
\Rightarrow-\lambda f(a)-\lambda A(u) \geqslant \frac{\rho}{2} .
\end{gathered}
$$

The final implication follows since $\lambda(s) \geqslant 0$. As in [7, Proposition 1.1] we choose $\beta=2$ and $\tau \in(0, \rho / 2)$ and let $\varepsilon=\rho / 2-\tau$. Now, by the continuity of $f$, there is $\delta_{1}>0$ such that

$$
\|f(x)-f(a)\|<\frac{\varepsilon}{2}
$$

whenever $\|x-a\|<\delta_{1}$. Also, since $f$ is locally Lipschitz about $a$ and $Y$ is reflexive it can be shown that, for some $\delta_{2}>0,\|A\| \leqslant k$ (where $k$ is the Lipschitz constant of $f$ about $a$ ) for each $A \in \partial f(x)$ provided $\|x-a\|<\delta_{2}$. Hence we shall assume

$$
\|x-a\|<\frac{\varepsilon}{4 k}=\delta_{3} \text {. }
$$

By the strong upper semi-continuity of the generalised Jacobian it follows that there is $\delta_{4}>0$ such that

$$
\partial f(x) \subseteq \partial f(a)+\frac{\varepsilon}{4} \mathcal{B}
$$


where $\mathcal{B}$ denotes the closed unit ball in $L(X, Y)$. Let $\delta=\min \left\{\delta_{i}, 1 / 2: i=1,2,3,4\right\}$. Now we let $U$ denote the open ball centred at $a$ of radius $\delta$.

Take any $x \in U \cap C$ and $\lambda \in S^{*}$ with $\|\lambda\|=1$, then there is $u \in(C-a) \cap X_{1}$ such that (6) holds. Set $v=u+a-x$ then, by the construction of $\delta, v \in(C-x) \cap X_{2}$. Now take any $\bar{A} \in \partial f(x)$, then there is $A \in \partial f(a)$ with $\|A-\bar{A}\|<\varepsilon / 4$ (by the strong upper semi-continuity). Hence, combining (6), (7), (8) and (9) we have

$$
\begin{aligned}
-\lambda(f(x)+\bar{A}(v)) & =-\lambda f(a)-\lambda A(u)+\lambda(f(a)-f(x))+\lambda(A-\bar{A})(u)+\lambda \bar{A}(u-v) \\
& \geqslant \frac{\rho}{2}-\frac{\varepsilon}{2}-\frac{\varepsilon}{4}-\frac{\varepsilon}{4}=\frac{\rho}{2}-\varepsilon=\tau>0 .
\end{aligned}
$$

Now the result follows by noting that, for any $\mu \in Y^{\prime}, \partial(\mu f)(x)=\mu \partial f(x)$. In addition, since $v \in(C-x) \cap X_{2} \subseteq T_{C}(x) \cap X_{2}$ it immediately follows that $v /\|v\| \in T_{C}(x) \cap X_{1}$.

REMARK. As a final remark on nonsmooth Robinson-type regularity conditions, recently the authors in [11] have shown, in the finite dimensional setting with $C=X$, that condition (2) is sufficient for local solvability of the constraint system. Here we assume that $f$ is locally Lipschitz and directionally differentiable at $a$, that is the limit

$$
f^{\prime}(a, d)=\lim _{t \downarrow 0} \frac{f(a+t d)-f(a)}{t}
$$

exists for each $d \in X$. Then the constraint system is locally solvable (see [4] and [11]) at $a$ if whenever $f(a)+f^{\prime}(a, d) \in-S$ has a solution $d \in X$ (with $\|d\|$ sufficiently small) there is a solution $x=a+\alpha d+o(\alpha)$ to $-f(x) \in S$ valid for all $\alpha>0$ sufficiently small. This constraint qualification has been used to establish asymptotic KKT conditions for quasidifferentiable programming problems. It is not difficult to adapt the argument used in [11] to show, under the assumptions of Theorem 4, that (URRC) is sufficient for local solvability. This result requires the metric regularity condition discussed above.

We complete our discussion of regularity conditions by showing that (RRC) is sufficient for the Karush-Kuhn-Tucker conditions. Consider (P) with a a minimum under the conditions of Theorem 3 assuming $C$ a closed convex set. Suppose that the multiplier $\tau=0$, hence there exists a nonzero $\lambda \in S^{*}$ such that

$$
0 \in \partial(\lambda f)(a)+N_{C}(a), \lambda f(a)=0
$$

Let us further suppose that $Y$ is reflexive and the regularity condition (RRC) is satisfied by the constraint system at $a$. By (10) there is a $\bar{A} \in \partial f(a)$ such that $-\lambda \bar{A} \in N_{C}(a)$ and $\lambda f(a)=0$. Now, by (RRC) and the generalised open mapping theorem, there is a positive constant $\rho_{0}$ such that

$$
Y_{p_{0}} \subseteq\left(\bar{A}\left(T_{C}(a) \cap X_{1}\right)+(S+f(a)) \cap Y_{1}\right)
$$


As in Theorem 4, let $D(A)$ denote the set on the right-hand side of (11) above. It follows by [24, Lemma 5.1] that $\sigma(\mu, D(A)) \geqslant \rho_{0}$ for each $\mu \in Y^{\prime}$ with $\|\mu\|=1$. If we take $\mu=-\lambda /\|\lambda\|$ and $\alpha>0$ with $0<\alpha<\rho_{0}$ then there is $y \in D(A)$ such that $-\lambda(y) \geqslant \alpha>0$. Hence, as $y=\bar{A}\left(x_{1}\right)+s+f(a)$ for some $x_{1} \in T_{C}(a) \cap X_{1}$, and $s \in S$, it follows that $-\lambda \bar{A}\left(x_{1}\right)-\lambda f(a) \geqslant \alpha>0$. (Here we note that $\lambda(s) \geqslant 0$.) However this is a contradiction to (10) since $\lambda \bar{A}\left(x_{1}\right) \geqslant 0$ (as $x_{1} \in T_{C}(a)$ and $\lambda f(a)=0$ ). Thus we must have $\tau>0$ whenever (RRC) holds at the minimum. It is clear, following a similar argument, that if $a$ is a regular point of the constraint system of $(P)$ then KKT conditions follow; however we are not assuming the uniform Robinson condition in the above. Hence this argument establishes the following KKT result:

THEOREM 5. For problem (P) assume that $h$ is locally Lipschitz, $f$ is locally compactly Lipschitzian and $C$ is a closed convex set. Furthermore assume $Y$ is a separable reflexive Banach space and that the constraint system satisfies (RRC) at $a$. Then a necessary condition for a to be a local minimum of $(P)$ is that there is $\lambda \in S^{*}$ such that $0 \in \partial h(a)+\lambda \partial f(a)+N_{C}(a)$ and $\lambda f(a)=0$.

CONCLUSION. We have shown that the class of locally compactly Lipschitzian mappings possess an important closedness condition in relation to a subgradient multifunction. This condition enables us to establish a chain rule, in either the separable or reflexive Banach space setting, and to restate a number of recently derived optimality conditions involving this class of mapping. In addition using a new concept of generalised Jacobian available in the infinite dimensional setting we are able to suggest an extension of the Robinson regularity condition to the infinite dimensional nonsmooth setting. This condition is shown to be sufficient for Karush-Kuhn-Tucker conditions to be established and is compared with existing regularity conditions. Following the recent work of Flam [10] it is possible to apply this result to stochastic programming.

\section{REFERENCES}

[1] J.M. Borwein, 'Stability and regular points of inequality systems', J. Optim. Theory Appl. 48 (1986), 9-52.

[2] J.V. Burke, 'An exact penalization viewpoint of constrained optimization', SIAM J. Control Optim. 29 (1991), 968-998.

[3] F.H. Clarke, Optimization and nonsmooth analysis (Wiley Interscience, New York, 1983).

[4] B.D. Craven, Mathematical programming and control theory (Chapman-Hall, London, 1978).

[5] P.H. Dien, 'Locally Lipschitzian set-valued maps and generalized extremal problems with inclusion constraints', Acta Math. Vietnam. 8 (1983), 109-122.

[6] P.H. Dien, 'On the regularity condition for the extremal problem under locally Lipschitz inclusion constraints', Appl. Math. and Optim. 13 (1985), 151-161. 
[7] P.H. Dien and N.D. Yen, 'On implicit function theorems for set-valued maps and their application to mathematical programming under inclusion constraints', Appl. Math. Optim. 24 (1991), 35-54.

[8] P.H. Dien and P.H. Sach, 'Further properties of the regularity of inclusion systems', Nonlinear Anal. 13 (1989), 1251-1267.

[9] I. Ekeland, 'On the variational principle', J. Math. Anal. Appl. 47 (1974), 324-353.

[10] S. Flåm, 'Lagrangian conditions and stochastic programming', SIAM J. Control Optim. 30 (1992), 1-10.

[11] B.M. Glover, V. Jeyakumar and W. Oettli, 'A Farkas lemma for difference sublinear systems and quasidifferentiable programming', School of Mathematics and Computing, Ballarat University College, Research Report 1/92, (submitted).

[12] R.B. Holmes, Geometric functional analysis and its applications (Springer-Verlag, Berlin, Heidelberg, New York, 1975).

[13] A.D. Ioffe, 'Regular points of Lipschitz mappings', Trans. Amer. Math. Soc. 251 (1979), 61-69.

[14] A. Jourani and L. Thibault, 'Approximate subdifferential and metric regularity: The finite dimensional case', Math. Programming 47 (1990), 203-218.

[15] H.-C. Lai, 'Optimality conditions for Lipschitz functions on Banach spaces', Nihonkai Math. Journal 2 (1991), 99-115.

[16] D. Ralph, Rank-1 support functionals and the generalized Jacobian, piecewise linear homeomorphisms, Ph.D Thesis (University of Wisconsin, Madison, 1990).

[17] S.M. Robinson, 'Stability theory for systems of inequalities, Part II: Differentiable nonlinear systems', SIAM J. Numer. Anal. 13 (1976), 497-513.

[18] P.H. Sach, 'Regularity, calmness and support principle', Optimization 19 (1988), 13-27.

[19] L. Thibault, 'Subdifferentials of compactly Lipschitzian vector-valued functions', Ann. Mat. Pura Appl. 125 (1980), 157-192.

[20] L. Thibault, 'On generalized differentials and subdifferentials of Lipschitz vector-valued functions', Nonlinear Anal. 6 (1982), 1037-1053.

[21] L. Thibault, 'Subdifferentials of nonconvex vector-valued functions', J. Math. Anal. Appl. 86 (1982), 319-354.

[22] N.D. Yen, Stability of the solution set of perturbed nonsmooth inequality systems, International Centre for Theoretical Physics (International Atomic Energy Agency, UNESCO, Trieste, Italy, 1990).

[23] K. Yosida, Functional analysis (Springer-Verlag, Berlin, Heidelberg, New York, 1980).

[24] J. Zowe and S. Kurcyuz, 'Regularity and stability of the mathematical programming problem in Banach spaces', Appl. Math. Optim. 5 (1979), 49-62.

School of Mathematics and Computing

Ballarat University College

Ballarat Vic 3353

Australia 